\title{
Long-term change in reactance by forced oscillation technique correlates with FEV1 decline in moderate COPD patients
}

\author{
To the Editor:
}

Since the landmark study of the natural history of chronic obstructive pulmonary disease (COPD) by Fletcher and Peto [1], lung function decline has been an issue of great concern and an important therapeutic target. The annual decline in forced expiratory volume in $1 \mathrm{~s}$ (FEV1) is generally used as a marker of disease progression. A review of large longitudinal studies found that the mean rate of FEV 1 decline was highest at Global Initiative for Chronic Obstructive Lung Disease (GOLD) stage II (47-79 $\left.\mathrm{mL} \cdot \mathrm{year}^{-1}\right)$, indicating the need for treatment at earlier stages of the disease [2].

The forced oscillation technique (FOT) is a noninvasive method that is used to measure respiratory system resistance $(R \mathrm{rs})$ and reactance $(X \mathrm{rs})$ during tidal breathing. Its clinical application has recently progressed worldwide with the spread of commercially available broadband frequency FOT devices. Because correlations between forced oscillatory parameters and spirometric indices are modest, complementary use of both tests may provide useful information regarding lung function [3]. In this study, we assessed whether there would be a long-term change in forced oscillatory parameters and whether the initial FOT index levels could predict the rate of change in these parameters, the so-called "horse-racing effect" [4], in a cohort of patients with COPD.

We retrospectively collected clinical data of outpatients who underwent FOT and spirometry on the same day at a 5-year interval at Shizuoka General Hospital (Shizuoka, Japan) between 2010 and 2015. Subjects included 24 male patients (mean values: age 75 years, body mass index $21.9 \mathrm{~kg} \cdot \mathrm{m}^{-2}$, smoking history 68 pack-years, FEV1 1.65 L and FEV1 z-score -3.18). All patients satisfied the GOLD definition of COPD and had been receiving medication, including long-acting muscarinic antagonists, long-acting $\beta_{2}$-agonists, inhaled corticosteroids or sustained-release theophylline. COPD grading was based on the GOLD classification and $\mathrm{FEV} 1 /$ height $^{2}\left(\mathrm{~L} \cdot \mathrm{m}^{-2}\right)$ [5]. The patients were clinically stable and had no exacerbations for $\geqslant 1$ month before the examination. Broadband frequency FOT and spirometry were performed using MostGraph-01 and CHESTAC-33 (Chest M.I., Tokyo, Japan), respectively. We used Rrs at 5 and $20 \mathrm{~Hz}$ ( $R 5$ and $R 20$, respectively) and the difference between $R_{5}$ and $R_{20}\left(R_{5}-R_{20}\right)$ as indicators of the frequency dependence of Rrs. In addition, we used $X_{\mathrm{rs}}$ at $5 \mathrm{~Hz}\left(X_{5}\right)$, which reflects elastic and inertial properties of the lung; resonant frequency $\left(F_{\mathrm{res}}\right)$, where $X_{\mathrm{rs}}$ crosses zero and the elastic and inertial forces are equal in magnitude and opposite; and a low-frequency reactance area (AX), which is an integral of $X_{5}$ to Fres. Oscillatory indices were expressed as mean values during a respiratory cycle. To determine pulmonary function while receiving daily treatment, medications were not withdrawn before FOT and spirometry, and measured data were expressed as post-bronchodilator values. FEV1 z-scores were derived from the Global Lung Function Initiative reference equations that adjust for age, sex, height and race [6]. The FOT indices were normally distributed after transforming into natural $\log (R 5, R 20$, Fres and $\mathrm{AX})$, square root $\left(R 5-R_{20}\right)$ or exponential (X5) values. Rrs and $X_{\mathrm{rs}} \mathrm{Z}$-scores were derived from the reference values of MostGraph measures for the middle-aged and elderly Japanese population $(n=784)$ who participated in annual health check-ups [7]. Comparisons between baseline and after 5 years were made using the paired t-test. Correlations between variables were performed using Pearson's correlation coefficient. A p-value of $<0.05$ was considered significant. EZR (version 1.27; Saitama Medical Center, Jichi Medical University, Saitama, Japan) was used for statistical analyses.

@ERSpublications

Respiratory system reactance changed over 5 years and the initial level predicted the rate of change in COPD patients http://ow.ly/ONzz308E3aD

Cite this article as: Akita T, Shirai T, Akamatsu T, et al. Long-term change in reactance by forced oscillation technique correlates with FEV1 decline in moderate COPD patients. Eur Respir J 2017; 49: 1601534 [https://doi.org/10.1183/13993003.01534-2016]. 
TABLE 1 Changes in forced expiratory volume in $1 \mathrm{~s}$ (FEV1) and respiratory system reactance ( $X$ rs ) indices over a 5-year interval

\begin{tabular}{|c|c|c|c|c|c|c|c|c|c|}
\hline & \multirow[t]{2}{*}{ Total } & \multicolumn{4}{|c|}{ GOLD classification } & \multicolumn{4}{|c|}{ FEV $1 /$ height $^{2}$ classification L.m ${ }^{-2}$} \\
\hline & & I & II & III & IV & $>0.5$ & $\leqslant 0.5$ to $>0.4$ & $\leqslant 0.4$ to $>0.3$ & $\leqslant 0.3$ \\
\hline Subjects $\mathrm{n}$ & 24 & 5 & 9 & 6 & 4 & 14 & 4 & 3 & 3 \\
\hline \multicolumn{10}{|l|}{ FEV1 z-score } \\
\hline Baseline & -3.18 & -0.55 & -2.48 & -4.52 & -6.05 & -1.82 & -4.18 & -5.22 & -6.19 \\
\hline After 5 years & $-3.56 *$ & -0.96 & $-3.25^{*}$ & -4.54 & -6.03 & $-2.38^{*}$ & -4.25 & -5.45 & -6.26 \\
\hline Change per year & -0.08 & -0.08 & -0.15 & -0.05 & 0.004 & -0.11 & -0.01 & -0.05 & -0.02 \\
\hline \multicolumn{10}{|l|}{$X_{5}$ z-score } \\
\hline Baseline & -1.06 & -0.33 & -0.45 & -1.62 & -2.48 & -0.41 & -1.55 & -1.95 & -2.50 \\
\hline After 5 years & $-1.53^{*}$ & -0.51 & $-1.28 *$ & -2.08 & -2.54 & $-1.01 *$ & -1.91 & -2.37 & -2.59 \\
\hline Change per year & -0.09 & -0.05 & -0.16 & -0.09 & -0.01 & -0.12 & -0.07 & -0.08 & -0.02 \\
\hline \multicolumn{10}{|l|}{ Fres z-score } \\
\hline Baseline & 1.18 & 0.45 & 0.42 & 1.81 & 2.87 & 0.45 & 1.52 & 2.41 & 2.94 \\
\hline After 5 years & $1.89 *$ & 0.80 & $1.45^{*}$ & 2.56 & 3.23 & $1.25^{*}$ & 2.12 & 3.05 & 3.42 \\
\hline Change per year & 0.14 & 0.07 & 0.21 & 0.15 & 0.07 & 0.16 & 0.12 & 0.13 & 0.10 \\
\hline \multicolumn{10}{|l|}{ AX z-score } \\
\hline Baseline & 1.00 & 0.37 & 0.40 & 1.53 & 2.34 & 0.40 & 1.35 & 1.95 & 2.40 \\
\hline After 5 years & $1.50 *$ & 0.59 & 1.17 & 2.01 & 2.60 & $1.00 *$ & 1.73 & 2.30 & 2.75 \\
\hline Change per year & 0.10 & 0.04 & 0.16 & 0.10 & 0.05 & 0.12 & 0.08 & 0.07 & 0.07 \\
\hline
\end{tabular}

Data are presented as mean, unless otherwise stated. GOLD: Global Initiative for Chronic Obstructive Lung Disease; $X_{5}$ : $X_{r s}$ at $5 \mathrm{~Hz}$; Fres: resonant frequency; $A X$ : low-frequency reactance area. ${ }^{*}$ : $p<0.05$ versus baseline.

FEV 1 and $X_{\mathrm{rs}}$ indices, including $X_{5}, F_{\mathrm{res}}$ and $\mathrm{AX}$, significantly changed at the 5-year interval (mean absolute values: FEV1 $1.65-1.40 \mathrm{~L}, X_{5}-1.48--2.08 \mathrm{cmH}_{2} \mathrm{O} \cdot \mathrm{L}^{-1} \cdot \mathrm{s}^{-1}$, Fres $12.34-15.87 \mathrm{~Hz}$ and $\mathrm{AX}$ 10.81-17.01 $\left.\mathrm{cmH}_{2} \mathrm{O} \cdot \mathrm{L}^{-1}\right)$. Changes in $\mathrm{FEV}_{1}$ and $\mathrm{Xrs} \mathrm{Z}$-scores by COPD grading system are shown in table 1. The changes per year for all parameters appear highest at GOLD stage II; however, this did not reach statistical significance. For the data graded by FEV1/height ${ }^{2}$, there is a trend for less annualised change in $X_{5}$, Fres and AX moving from group 1 through to group 4, which is not seen in the GOLD classification. There were no significant changes in $R$ rs indices, including $R 5, R 20$ and $R 5-R 20$ over 5 years. There was a significant correlation between change in FEV1 and $X_{r s}$ (correlation coefficient: X5 0.410, Fres 0.422 and $\mathrm{AX}$ 0.434). Concerning the horse-racing effect, there was no correlation between the initial FEV1 level and the rate of change in FEV1, regardless of whether analysed by absolute value or z-score. However, there was a negative correlation between the initial $X \mathrm{rs}$ Z-scores and the rate of change in $X_{\mathrm{rs}}$ z-scores (correlation coefficient $X_{5}-0.546, F_{\text {res }}-0.411$ and AX -0.591 ). No correlation was observed between the initial absolute $X$ rs levels and the rate of change in absolute Xrs.

In this study, $X_{\mathrm{rs}}$ indices changed significantly over 5 years, and there was a significant correlation between changes in FEV 1 and $X$ rs. This is the first study to indicate that the lung function decline in patients with COPD affects not only spirometry but also FOT. This suggests that $X_{\mathrm{rs}}$ indices, including $X 5$, Fres and AX, measured during tidal breathing, are markers of lung function decline in patients with COPD. The lack of significant changes in Rrs indices over 5 years may be explained by the previous finding that $X_{r s}$ indices were more sensitive to the increased severity of COPD than Rrs indices [8]. The relationship between the FEV 1 level and the rate of change in FEV 1 has been termed the horse-racing effect, meaning that the lower the level of function, the greater the rate of decline [4]. In this study, we found a negative correlation between the initial $X \mathrm{rs} \mathrm{Z}$-scores and the rate of change in $X \mathrm{rs} \mathrm{Z}$-scores, suggesting that the measurement of $X \mathrm{rs}$ leads to the early detection of lung function decline in patients with COPD. The lack of significant correlation between the FEV1 level and the rate of change in FEV1 could potentially be explained by the limited sample size. Altogether, the results obtained by FOT correlate with those obtained by spirometry, but they are not identical. This indicates that FOT is not a surrogate test for spirometry, but should be used concomitantly. In older patients who have difficulty in undergoing a spirometry test, FOT would be particularly useful. Additional larger prospective studies are required to confirm whether $\mathrm{Xr}_{\mathrm{r}}$ is useful as a marker of lung function decline and can be used as a therapeutic target in COPD.

Takefumi Akita $^{1,2}$, Toshihiro Shirai ${ }^{1,2}$, Taisuke Akamatsu ${ }^{1}$, Mika Saigusa ${ }^{1}$, Akito Yamamoto $^{1}$, Yuichiro Shishido ${ }^{1}$, Satoru Morita ${ }^{1}$ and Kazuhiro Asada ${ }^{1}$

${ }^{1}$ Dept of Respiratory Medicine, Shizuoka General Hospital, Shizuoka, Japan. ${ }^{2}$ Both authors contributed equally. 
Correspondence: Toshihiro Shirai, Shizuoka General Hospital, 4-27-1 Kita-Ando, Aoi, Shizuoka 420-8527, Japan.

E-mail: toshihiro-shirai@i.shizuoka-pho.jp

Received: April 062016 | Accepted after revision: Jan 202017

Conflict of interest: None declared.

\section{References}

1 Fletcher C, Peto R. The natural history of chronic airflow obstruction. Br Med J 1977; 1: 1645-1648.

Tantucci C, Modina D. Lung function decline in COPD. Int J Chron Obstruct Pulmon Dis 2012; 7: 95-99.

Shirai T, Kurosawa H. Clinical application of the forced oscillation technique. Intern Med 2016; 55: 559-566.

Burrows B, Knudson RJ, Camilli AE, et al. The "horse-racing effect" and predicting decline in forced expiratory volume in one second from screening spirometry. Am Rev Respir Dis 1987; 135: 788-793.

5 Miller MR, Pedersen OF, Dirksen A. A new staging for chronic obstructive pulmonary disease. Int J Chron Obstruct Pulmon Dis 2007; 2: 657-663.

6 Quanjer PH, Stanojevic S, Cole TJ, et al. Multi-ethnic reference values for spirometry for the 3-95-yr age range: the global lung function 2012 equations. Eur Respir J 2012; 40: 1324-1343.

7 Abe Y, Shibata Y, Igarashi A, et al. Reference values of MostGraph measures for middle-aged and elderly Japanese individuals who participated in annual health checkups. Respir Investig 2016; 54: 148-155.

8 Di Mango AM, Lopes AJ, Jansen JM, et al. Changes in respiratory mechanics with increasing degrees of airway obstruction in COPD: detection by forced oscillation technique. Respir Med 2006; 100: 399-410.

Copyright @ERS 2017 